\title{
EREBEA
}

Revista de Humanidades

y Ciencias Sociales

NúM. 3 (2013), pp. 103-116

ISSN: 0214-0691

\section{HiSTORIE AUTORISÉE, BIOGRAPHIE HÉROÏSÉE: LES DOUZE TRIOMPHES DE HENRY VII (I 497)*}

\author{
Gilles Lecuppre \\ Université Paris Ouest-Nanterre-La Défense
}

Resumen

Merced a un singular poema francés, cuyos orígenes siguen siendo oscuros, el rey Tudor Enrique VII se transformó en 1497 en un segundo Hércules y parte de su biografía, en una sucesión de doce trabajos mitológicos. Desde luego, Enrique aparecía descrito con harta complacencia, aventajando a su modelo grecorromano y derribando a peores adversarios que monstruos de la Antigüedad.

Por desgracia, esta pretenciosa y lúdica obra daba a entender al lector que la tarea no se había llevado totalmente a buen término y que el usurpador, pese a toda su gallardía y virtud, apenas lograba amilanar a sus enemigos ni a ganarse el corazón de sus súbditos. ¿De aquí, quizás, el malogro final de esta asombrosa biografía heroica?
Abstract

Thanks to a singular French poem, whose origins remain obscure, the Tudor king Henry VII was turned in 1497 into a second Hercules, and part of his biography thus became a succession of twelve mythological labours. Henry was complacently shown as surpassing his Greco-Roman model and as slaying opponents who were worse than the monsters of antiquity.

Unfortunately, the pedantic and playful work led its reader to understand that the task was not successfully finished, and that the usurper, in spite of all his bravery and virtue, could hardly discourage his enemies, or even win the hearts of his subjects. Hence, perhaps, the final failure of that amazing heroic biography?

\section{KEYWORDS \\ Henry VII, Biography. Mythology.}

Fecha de recepción: 1 de julio de 2013 Fecha de aceptación: 31 de julio de 2013

* Je tiens ici à exprimer toute ma reconnaissance à D. Javier Pérez-Embid, qui a accueilli avec bienveillance cette contribution exotique dans ce numéro de la revue, ainsi qu’à Manuela Águeda García-Garrido, qui a traduit en espagnol le résumé de cet article avec gentillesse et talent. 

Tout au long du Moyen Âge, Hercule a été christianisé, moralisé, travesti en philosophe, en femme ou en chevalier ${ }^{1}$. Au XV siècle, au terme de ces multiples métamorphoses, le héros se met au service des princes : il prête sa fermeté d'âme à la famille d'Este, fonde rétrospectivement Florence et parraine les Médicis, ou se raccroche à la généalogie des ducs Valois de Bourgogne 2 . On considère fréquemment que les dernières années du siècle sont les plus propices au rapprochement entre les rois les plus magnifiques et le héros à la croisée des chemins. Il est vrai que Matthias Corvin, Charles VIII, puis Maximilien de Habsbourg ont fait l'objet de parallèles flatteurs avec Hercule, mais de manière beaucoup plus ténue qu'on ne serait tenté de le croire ${ }^{3}$. Dans ce discret concert européen, les Douze triomphes de Henry VII forment par contraste une œuvre de quelque importance, puisque ce poème en français, certes conservé par un seul manuscrit, comprend 632 vers en décasyllabes, avec le schéma des rimes abab$b c b c^{4}$. Ce qui frappe surtout est le caractère systématique de l'entreprise, qui enferme la première moitié du règne du roi d'Angleterre Henri VII, fondateur de la dynastie Tudor, dans le cadre strict et presque classique des douze travaux. La biographie royale et la chronique de son temps tout à la fois sont héroïsées. L'histoire du royaume et la vie du monarque sont portées sur un même plan

1 Un bilan par le grand connaisseur : M.-R. Jung, " Hercule dans les textes du Moyen Âge : essai d'une typologie ", dans A. M. ВАвв (ed.) : Rinascite di Ercole. Convegno internazionale. Verona, 29 maggio-1 giugno 2002, Vérone : Fiorini, "Medioevi Studi », 2002, p. 9-69.

2 A. Tissoni Benvenuti, "Il mito di Ercole. Aspetti della ricezione dell'antico alla corte Estense nel primo Quattrocento ", dans Omaggio a Gianfranco Folena, Padoue : Editoriale Programma, 1993, p. 773-792. A. Wright, "The myth of Hercules", dans G. C. Garfagnini (ed.) : Lorenzo il Magnifico e il suo mondo, Leo S. Olschki Editore, 1994, p. 323-339. M. ChEYns-Condé, "L'adaptation des "Travaux d'Hercule" pour les fêtes du mariage de Marguerite d'York et de Charles le Hardi à Bruges en 1468 ", dans Publications du Centre Européen d'Études Bourguignonnes, vol. 34 (1994), p. 71-85 ; A. Millar, "Olivier de la Marche and the Herculean origins of the Burgundians, dans Publications du Centre Européen d'Études Bourguignonnes, vol. 41 (2001), p. 67-75.

3 V. Rees, "Transformation and self-fashioning: Matthias Corvinus and the myth of Hercules ", dans Annual of Medieval Studies at CEU, vol. 11 (2005), p. 167-186. G. BraungarT, « Mythos und Herrschaft : Maximilian I. als Hercules Germanicus ", dans W. Haug et B. Wachinger (eds.) : Traditionswandel und Traditionsverhalten, , Tübingen : Niemeyer, 1991, p. 77-95.

4 Les Douze triomphes de Henry VII, dans J. Gairdner (ed.) : Memorials of King Henry the Seventh, Londres : Longman, Rerum Britannicarum Medii Aevi Scriptores, "Rolls series », 10, 1858, p. 133-153 (avec traduction anglaise p. 307-327). 
mythique, qui brouille la chronologie et exalte la valeur incommensurable d'un être surhumain.

On peut parler néanmoins à l'endroit de cette production originale, dans une certaine mesure, de poème maudit, relativement négligé par les historiens de la littérature et du politique. Plusieurs raisons concourent sans doute à cette désaffection : sa situation dans une période charnière, qui pousse médiévistes et modernistes à une prudence excessive, son appartenance à un genre encomiastique longtemps tenu pour insignifiant, sa langue même qui le place en porte à faux. Les historiens de l'Angleterre ont préféré exploiter le rapport plus substantiel des chroniques ${ }^{5}$, tandis que l'œuvre échappait même à l'attention de Marc-René Jung, grand connaisseur des destinées herculéennes ${ }^{6}$. Pour comble d'infortune, son attribution elle-même est devenue problématique. Son éditeur, James Gairdner, y reconnaissait en 1858 la paternité de Bernard André sur la foi de la langue, du style, de la cohérence avec les images employées par ailleurs. Né à Toulouse vers 1450, frère augustin, docteur dans les deux droits, arrivé en Angleterre juste après l'instauration de la nouvelle monarchie avec l'intention de se spécialiser dans l'éloge de ses exploits, il en obtient dons et pensions et partage son œuvre entre chroniques et annales, d'une part, et poésie panégyrique ou religieuse en latin et plus rarement en français, de l'autre. Apprécié, parmi d'autres, par Henri VII et Henri VIII, il jouit du statut de poète-lauréat et se voit un temps confier la tutelle du fils aîné du premier, Arthur?. Sa position de chantre quasi officiel versé dans la mythologie combinée à sa cécité font de lui l'Homère du Nord, vers qui convergent les diverses influences littéraires de son temps. En 1998, toutefois, David Carlson, répertoriant les œuvres de Bernard André, a préféré classer les Douze triomphes parmi les pièces d'origine incertaine, en arguant du fait que bien d'autres Français lettrés gravitaient autour du roi d'Angleterre en 1497, date de sa composition ${ }^{8}$. Cette réserve, à mon sens, n'invalide pas totalement le raison-

5 La biographie de référence reste celle de S. B. Chrimes, Henry VII, Londres : Yale University Press, Yale English Monarchs Series, 1999.

$6 \mathrm{Ni}$ le répertoire évoqué plus haut, ni son œuvre maitresse n'y font allusion (M.-R. JunG, Hercule dans la littérature française du XVI siècle : de l'Hercule courtois à l'Hercule baroque, Genève : Droz, "Travaux d'humanisme et Renaissance ", 1966).

7 Outre l'introduction de Gairdner, voir G. Tournoy, "Bernard André ", dans P. G. BietenhOlz et T. B. Deutscher (eds.) : Contemporaries of Erasmus. A Biographical Register of Renaissance and Reformation, Toronto/Buffalo/Londres : University of Toronto Press, 1985, p. 52-53. L'auteur annonçait alors une étude majeure du personnage à l'initiative de Constance Blackwell qui, à ma connaissance, n’a pas été publiée.

8 D. R. Carlson, "The writings of Bernard André (c. 1450-c. 1522)", dans Renaissance Studies: Journal of the Society for Renaissance Studies, vol. 12:2 (1998), p. 229-250. Quelques années plus tard, un nouveau repertoire oublie totalement le panégyrique : D. HоввINs, «Arsenal Ms 360 as a witness to the career and writings of Bernard André ", dans Humanistica Lovaniensia: Journal of Neo-Latin Studies, vol. 50 (2001), p. 161-198. 
nement de Gairdner, mais contribue certainement à affaiblir la curiosité pour le poème.

Laissons là momentanément cette question de l'auteur pour analyser un peu plus en détail ces vers qui attestent le renouveau des goûts et des supports de l'expression en s'attelant à la mise en forme mythique du parcours d'un roi besogneux. La démarche, pédante et ludique, peut s'apparenter, comme nous l'avons dit, à la réalisation d'une chronique poétique du règne, ce qui retiendra notre attention dans un premier temps. Au cœur du défi se trouve la lourde comparaison du héros et du roi, aboutissant en somme à une hérö̈sation du monarque. Pour Henri VII comme pour Hercule, cependant, la liste des travaux pourrait bien ne pas être close et le fardeau du pouvoir semble, en ces temps de tribulations, plus que jamais requérir au minimum la force et la fortune d'un demi-dieu.

Pour la commodité de l'exposé, j'ai fait figurer ci-dessous un tableau comparatif des aventures du roi et de son modèle, qui permettra une circulation plus facile dans les épisodes de leur vie sélectionnés et recomposés par le poète.

\section{LES DOUZE TRIOMPHES DE HENRY VII}

\section{SCHÉMA GÉNÉRAL}

Junon, par l'intermédiaire d'Eurysthée, veut faire perdre la vie à Hercule en lui imposant des travaux périlleux.

L'Envie (= la "douairière de Flandre ", Marguerite d'York), avec l'aide de " celui qui se dit roi des Romains" (= Maximilien), soumet le roi Henri VII à des épreuves redoutables et incessantes.

\section{LES TRAVAUX}

\begin{tabular}{|c|l|l|}
\hline 1 & $\begin{array}{l}\text { Hercule vainc le lion Cleonus, puis } \\
\text { se vêt de sa peau, qui le protège } \\
\text { jusqu'à sa mort. }\end{array}$ & $\begin{array}{l}\text { Henri vainc le roi superbe et noble } \\
\text { par-delà la mer (= le roi de France } \\
\text { Charles VIII ?). Sa peau = les ver- } \\
\text { tus propres aux rois : force, pru- } \\
\text { dence, richesse. }\end{array}$ \\
\hline 2 & $\begin{array}{l}\text { Hercule tue l'Hydre de Lerne, un } \\
\text { lac d'où jaillissent des ruisseaux qui } \\
\text { dévastent le pays et que le héros } \\
\text { parvient à assécher. }\end{array}$ & $\begin{array}{l}\text { Henri lutte contre Envie } \\
\text { (Marguerite d'York), sale, dépe- } \\
\text { naillée et venimeuse. Il assèche } \\
\text { les mauvais courants de la guerre } \\
\text { civile. }\end{array}$ \\
\hline 3 & Hercule tue le sanglier d'Arcadie. & $\begin{array}{l}\text { Henri tue le roi Richard (Richard } \\
\text { III), incarnation de l'avarice et du } \\
\text { mal. }\end{array}$ \\
\hline
\end{tabular}




\begin{tabular}{|c|c|c|}
\hline 4 & Hercule tue le cerf aux cornes d'or. & $\begin{array}{l}\text { Henri tue le comte de Licaon (= } \\
\text { John de la Pole, comte de Lincoln), } \\
\text { venu en ce pays, aidé des soudards } \\
\text { d'Envie, pour détruire le roi. }\end{array}$ \\
\hline 5 & $\begin{array}{l}\text { Hercule tue et chasse de son arc, } \\
\text { jusqu'au dernier, les oiseaux du } \\
\text { lac Stymphale, qui mangeaient } \\
\text { hommes et bêtes. }\end{array}$ & $\begin{array}{l}\text { Henri chasse, au moyen de sa jus- } \\
\text { tice, les larrons qui régnaient sur } \\
\text { mer comme sur terre. }\end{array}$ \\
\hline 6 & $\begin{array}{l}\text { Hercule se saisit de la ceinture de } \\
\text { Ménalipe, reine des Amazones. }\end{array}$ & $\begin{array}{l}\text { La douairière de Flandre et de } \\
\text { Bourgogne (Marguerite d'York) } \\
\text { s'est privée inutilement de ses ri- } \\
\text { chesses en faisant couronner un } \\
\text { coquin en Irlande. }\end{array}$ \\
\hline 7 & $\begin{array}{l}\text { Hercule vient à bout de Diomède et } \\
\text { de ses juments anthropophages. }\end{array}$ & $\begin{array}{l}\text { Henri a taillé en pièces Martin } \\
\text { Swart (Martin Schwarz) sur le } \\
\text { champ de bataille. }\end{array}$ \\
\hline 8 & $\begin{array}{l}\text { Hercule terrasse le taureau [de } \\
\text { Crète]. }\end{array}$ & $\begin{array}{l}\text { Henri soumet par son habileté le } \\
\text { roi d'Écosse (= Jacques } I V) \text { et ses } \\
\text { gens. }\end{array}$ \\
\hline 9 & $\begin{array}{l}\text { Hercule vainc Géryon aux trois têtes, } \\
\text { possesseur de grands troupeaux. }\end{array}$ & $\begin{array}{l}\text { Henri n'a pas été détruit par la } \\
\text { triple attaque du roi des Romains } \\
\text { (Maximilien), de l'archiduc [d'Au- } \\
\text { triche] (Philippe le Beau) et de la } \\
\text { douairière (Marguerite d'York). }\end{array}$ \\
\hline 10 & $\begin{array}{l}\text { Cacus, grand larron dont la bouche } \\
\text { crache le feu, essaie de dérober } \\
\text { à Hercule les boufs de Géryon. } \\
\text { Hercule le chasse par sa vaillance. } \\
\text { Cacus s'enfuit dans une caverne } \\
\text { peuplée d'autres brigands. }\end{array}$ & $\begin{array}{l}\text { Pietrequin (Perkin Warbeck) n'a } \\
\text { pas su garder ses soudards, en } \\
\text { dépit des menaces qu'il proférait. } \\
\text { Henri l'a fait fuir en Irlande avec } \\
\text { les sauvages. }\end{array}$ \\
\hline 11 & $\begin{array}{l}\text { Hercule emmène Cerbère, monstre } \\
\text { aux trois têtes, gardien du marais } \\
\text { des enfers. }\end{array}$ & $\begin{array}{l}\text { Henri punit trois capitaines } \\
\text { - Jennot (Genyn), Quentin } \\
\text { (Quyntyne) et Beld (John Belt). }\end{array}$ \\
\hline 12 & $\begin{array}{l}\text { Henri vainc le dragon Maxile pour } \\
\text { cueillir les pommes d'or du jardin des } \\
\text { Hespérides. }\end{array}$ & $\begin{array}{l}\text { Henri vainc Maximilien, qui } \\
\text { gardait la porte pour l'empêcher } \\
\text { d'avoir l'amour du noble roi de } \\
\text { France (Charles VIII). }\end{array}$ \\
\hline
\end{tabular}




\section{I) L'histoire ET LA BIOGRAPHIE STRUCTURÉES PAR LE MYTHE}

Les Douze triomphes re-déploient symboliquement les douze premières années du règne du Tudor. Ils proposent en quelque sorte un équivalent versifié et réordonné de la vie de Henri VII par Bernard André, qui assume également l'année 1497, certes poussée un peu plus avant, comme son terme ${ }^{9}$. Pour le poète, sans nul doute, le mythe prime l'histoire. En historien pondéré, je propose pour commencer de rétablir la chronologie bouleversée, ce qui nous permettra d'ailleurs de comprendre certains des mécanismes qui président au va-et-vient entre les travaux du héros et les exploits de l'usurpateur.

Chacun sait que la couronne d'Angleterre a été ramassée par Henri sur le champ de bataille de Bosworth le 22 août 1485, aux dépens de Richard III. En ce sens, la première épreuve du roi correspond à la troisième rencontrée par Hercule, celle du sanglier d'Érymanthe, que le poème appelle simplement sanglier d'Arcadie $^{10}$. L'association du tyran Richard III, assassin de ses deux neveux, avec le sanglier est naturelle puisque lui-même s'était donné l'animal pour devise ("un sanglier marchant d'argent, défendu, hérissonné et ancorné d'or "). Le poète fait mine de s'étonner de ce choix, qu'il explique par la somme des péchés que représente traditionnellement le sanglier, à laquelle il ajoute du reste la mention plus originale de l'avarice ${ }^{11}$. Appelé de son vivant the Boar ou the Hog, c'est-à-dire le Sanglier et le Pourceau ${ }^{12}$, cet homme perclus de vices était voué à la mort brutale qu'exigeait la justice divine, au prix d'un petit écart avec la version originelle du mythe, où Hercule capturait seulement la bête.

L'installation du roi Henri n'a pas été des plus faciles. Dès 1487, un complot de grande ampleur vise à le déloger. Il est en réalité centré sur un jeune imposteur, Lambert Simnel, que les conspirateurs tiennent pour Édouard de Warwick, neveu des deux précédents rois de la dynastie d'York ${ }^{13}$. Il n'est pas cité ici, l'accent étant placé, aux $4^{\mathrm{e}}$ et $7^{\mathrm{e}}$ gestes, sur les bras armés de la manœuvre. Le premier en est le cerf aux cornes d'or, biche aux pieds d'airain virilisée pour la circonstance,

9 Bernardi Andreae Vita Henrici Septimi, dans GaIrdner (ed.) : Memorials of King Henry... p. 3-75.

10 Les Douze triomphes de Henry VII, p. 138-139.

11 Citons par exemple M. PAstoureau, «Chasser le sanglier. Du gibier royal à la bête impure: histoire d'une dévalorisation ", dans Une Histoire symbolique du Moyen Âge occidental, Paris : Seuil, 2004, p. 65-77.

12 Un gentilhomme du Wiltshire fit d'ailleurs les frais de quelques vers de son cru, où il raillait le roi et ses conseillers, William Catesbie, Richard Ratcliffe et Lord Lovell, en leur donnant des noms d'animaux : "The Catte, the Ratte and Louell our dogge Rulyth all Englande under a hogge ». Voir notamment J. G. Bellamy, The Law of Treason in England in the Later Middle Ages, Cambridge (Mass.) : Cambridge University Press, rééd. 2004, p. 121-122.

13 M. J. Bennetr, Lambert Simnel and the Battle of Stoke, Stroud : Sutton, 1987. 
John de la Pole, comte de Lincoln, héritier présomptif de Richard III ${ }^{14}$. Ayant trompé la bienveillance de son nouveau maître, Henri VII, il rejoint quelques représentants de l'aristocratie anglaise et irlandaise pour accompagner le petit roi qu'ils couronnent à Dublin, mener un débarquement et une marche de grand style, misérablement achevée sur le champ de bataille d'East Stoke le 16 juin 1487. Perfide et subtil, le poète transforme Lincoln en Licaon, nom qui évoque un personnage de la mythologie célèbre pour avoir servi de la chair humaine à Zeus. Son partenaire dans le crime est le mercenaire allemand Martin Schwarz ${ }^{15}$, brute arrogante qui avait autrefois paradé dans Bruxelles en roi conquérant et dont les princes des Pays-Bas avaient détourné les ravages au profit de la singulière cause yorkiste. Tourné en ridicule en Diomède, propriétaire de chevaux anthropophages, il est stigmatisé pour sa cruauté et ses fanfaronnades, qui lui ont valu et lui vaudront par ailleurs d'évoluer en croque-mitaine dans divers proverbes et chansons de ce temps ${ }^{16}$.

Plus anecdotique est au fond l'épisode de 1492, malgré sa position liminaire et le voile très transparent dont le poète feint de couvrir le protagoniste. Le lion Cleonus ou de Némée cache un roi superbe "Et de ce roy je me taiz le nommer,/ Qui du leon est icy figuré ${ }^{17}$. Il suffit de savoir qu'il a failli dévorer Henri quand il était " delà la mer " pour reconnaître, à la suite de James Gairdner, le roi de France Charles VIII, désormais ami des Anglais, qui fit l'objet d'une vague expédition de Henri VII, réglée par la paix d'Étaples, le 6 novembre 1492. La nouvelle entente cordiale impose la réserve; le souvenir glorieux exige qu'on la transgresse ${ }^{18}$.

Une seconde cabale plutôt respectable, évoquée par quatre travaux, agita les années 1490. De nouveau, un faux prince d'York, Perkin Warbeck, originaire de Tournai, rallia les suffrages des mécontents et obtint quant à lui le soutien d'une part significative des rois occidentaux ${ }^{19}$. Ainsi se lit la capture de Cerbère ${ }^{20}$, monstre tricéphale désignant trois capitaines que je pense être le premier à avoir

14 Les Douze triomphes, p. 139-140.

15 Les Douze triomphes, p. 142-143.

16 William Wager, dans une pièce morale allégorique de 1569, fait entrer sur scène l'incarnation d'une jeunesse dérangée, Moros, qui entame une série d'airs populaires, dont "Martin Swart and his man, sodledum, sodledum / Martin Swart and his man, sodledum bell " (W. WAGER, The Longer thou Livest, R. M. Benвow (ed.), Londres : Edward Arnold, 1978, p. 7). L'homme de Swart n'est autre que sa marionnette, Lambert Simnel.

17 Les Douze triomphes, p. 135-136.

18 Pour une comparaison avec une autre héroïsation classicisante de cet épisode : D. R. CARLson, "The Italian Johannes Opicius on Henry VII's invasion of France : historical witness and antique convention ", dans Renaissance Studies: Journal of the Society for Renaissance Studies, 20:4 (2006), p. 520-546.

19 I. Arthurson, The Perkin Warbeck Conspiracy. 1491-1499, Stroud : Sutton, 1997. Sur le phénomène de l'imposture dans toutes ses dimensions, G. Lecuppre, L'Imposture politique au Moyen Âge. La seconde vie des rois, Paris : PUF, 2005.

20 Les Douze triomphes, p. 147-148. 
pris la peine de reconnaître. Jennot, Quentin et Beld, ou plutôt Genyn, mercenaire breton, Quyntyne, espagnol et Belt, anglais, étaient trois cadres de la troupe débarquée au nord de Deal, le 3 juillet 1495, pour servir de tête de pont à l'expédition menée au nom de Warbeck, alias Richard d’York. Défaits par les troupes royales de Sandwich, les rebelles survivants furent traités comme des pirates et décapités quelques jours plus tard ${ }^{21}$. Ils incarnent, de manière abusive dans le poème, la trahison et son juste châtiment. Parmi les princes tactiquement séduits par la subversion yorkiste, Jacques IV d'Écosse n'est pas le moindre, qui mena en septembre 1496 un raid assez stérile contre le nord de l'Angleterre, payé de retour par une riposte anglaise moins d'un an plus tard, rapidement suivie d'une paix entre les deux royaumes, ce que résume le $8^{\mathrm{e}}$ travail, qui voit Hercule dompter le taureau de Crète ${ }^{22}$. L'épopée de Warbeck, lâché par l'Écosse, ne prenait pas fin pour autant. Le jeune homme suscite la $10^{\mathrm{e}}$ prouesse : là où Cacus, larron cracheur de feu, avait tenté de voler à Hercule une partie des troupeaux qu'il avait raflés à Géryon, le pseudo-prince a voulu faire main basse sur des hommes de guerre étrangers, qu'il a perdus en route, voire sur le royaume, que ses paroles incendiaires menaçaient ${ }^{23}$. Mis en déroute, à l'instar de son prédécesseur mythologique, il s'est réfugié en un lieu ô combien inhospitalier : "Ce feu gettant, c'estoit son menacer,/ Car il pensoit faire de grans outrages ; /Mais le bon roy le fist tantost cacher/ En Yrlande avecque les sauvages ». Toute cette aventure, encore inachevée, a été rendue possible par le concours d'un trio maléfique, les trois têtes de Géryon, Maximilien, que le poète s'acharne à appeler « roi des Romains » alors qu'il est empereur de plein exercice, son fils Philippe le Beau, archiduc d'Autriche et la douairière de Bourgogne, veuve de Charles Téméraire, Marguerite d'York, sœur des deux rois Édouard IV et Richard III ${ }^{24}$.

De même que la succession des travaux a une seule origine, la haine inextinguible de Junon pour Hercule, mis en danger sur l'ordre de la déesse par le pusillanime Eurysthée, les malheurs du roi Henri sont tout entiers dus à la terrible Marguerite, dont le gendre, Maximilien, est l'Eurysthée servile et secondaire ${ }^{25}$. Il est remarquable que Bernard André ait qualifié Marguerite de Junon dans ses œuvres historiques et que ce haro sur une instigatrice unique et plus facile à isoler corresponde à la ligne diplomatique définie par le monarque et par ses conseillers : faire peser la responsabilité sur cette vieille acariâtre aux ressources limitées et dédouaner les autres grands souverains de leur culpabilitée ${ }^{6}$. Dès le

21 The Great Chronicle of London, A. H. Thomas et I.D. Thornley (eds.), Londres : George W. Jones, 1938, p. 205-206.

22 Les Douze triomphes, p. 143-144.

23 Les Douze triomphes, p. 146-147.

24 Les Douze triomphes, p. 144-145.

25 Les Douze triomphes, p. 134.

26 On trouve ainsi Marguerite en Junon dans Bernardi Andreae Vita Henrici Septimi, p. 65. 
prologue, la douairière est peinte en Junon, ou encore en allégorie de l'Envie ou Fausse Envie ${ }^{27}$. La jalouse traîtresse est apostrophée ici ou là pour avoir alimenté de ses troupes les tentatives d'invasion. Polymorphe, sinon protéiforme, elle prend part directement à trois travaux, au prix de la cohésion poétique : d'abord hydre "Orde et salle, villement acoultrée, getant venyn sur grans et petis ${ }^{28}$, elle revient sous les traits a priori plus flatteurs de la reine des Amazones, ici nommée Ménalipe, dont Hercule a volé la ceinture ${ }^{29}$, puis devient une des têtes du formidable Géryon, métaphore d'une alliance des princes des Pays-Bas ${ }^{30}$. Sa laideur est morale, sa richesse réelle mais gaspillée dans de folles manigances ; elle serait comique sans sa constance dans le mal. Tous les autres ne sont que des comparses, parmi lesquels Maximilien, géant ou dragon de papier, demeure incapable et sans gloire après son vain labeur.

A contrario, vainqueur de tous les périls envoyés par Envie, Henri joue, mais sur un mode mineur, ce rôle de civilisateur que l'on prête parfois au héros gréco-romain. De son feu de charité, il a asséché les ruisseaux dévastateurs de la guerre civile ${ }^{31}$. De sa justice, il a mis fin au brigandage sur terre comme sur mer ${ }^{32}$. L'amour retrouvé du roi de France a rendu la possibilité aux bons marchands de circuler entre les deux pays. Ces maigres acquis sont fort contestables. L'auteur passe évidemment sous silence des faits très déplaisants, tels que l'initiative française dans l'équipée de Perkin Warbeck ou l'interruption éminemment dommageable au commerce anglais du Magnus Intercursus, la relation maritime avec la Flandre $^{33}$. Mais c'est surtout dans l'économie de son propos qu'il apparaît que les victoires du nouveau roi sont minimes, partielles ou fragiles, comme nous verrons plus loin.

\section{II) LE HÉROS ET LE ROI}

Les portraits croisés du héros et du roi obéissent à une esthétique qui s'efforce de tourner le dos à celle qui accompagnait les occurrences antérieures d'Hercule, mais aussi et surtout à un projet rhétorique visant à confondre les deux personnalités, pour mieux exalter la supériorité du dédicataire (ou du commanditaire).

Dès la troisième strophe, le poète dévoile son intention de se plier à la mode. Il lui faut, dit-il, une figure, une comparaison puisée dans "l'histoire des Grecs ", pour chanter correctement les hauts faits et les triomphes du souverain persé-

27 Les Douze triomphes, p. 133-135.

28 Les Douze triomphes, p. 137.

29 Les Douze triomphes, p. 141-142.

30 Les Douze triomphes, p. 145.

31 Les Douze triomphes, p. 138.

32 Les Douze triomphes, p. 141.

33 Le texte de l'embargo : Tudor Royal Proclamations, P. L. Hughes et J. F. Larkin (eds.), vol. 1, New Haven et Londres : Yale University Press, 1964, p. 35. 
cuté $^{34}$. Ses investigations l'ont mené vers l'envie de Junon contre Hercule, qui dessine le sujet le plus approchant. À la différence de nombre de ses devanciers, il peut se piquer de pouvoir livrer la liste classique des travaux, sans doute puisée dans la Bibliothèque de Diodore traduite par le Pogge, à l'exception notable du Cacus très romain, vraisemblablement substitué pour des raisons de décence aux écuries d'Augias. Sa culture mythologique, attestée par l'allusion à Licaon, se double d'interprétations étymologiques ou historiques utiles aux contorsions qui lui font atteindre la personnalité moderne visée. Par exemple, "Ysdra, c’est Grec qui vault autant à dire/En bon latin aqua: ce sont deux eaux ${ }^{35}$. L'idée du lac qui déborde et dévaste prend le pas sur celle du serpent et fournit l'occasion de canaliser les énergies destructrices de la guerre des Deux-Roses. Selon un procédé voisin, il prépare la notion de parenté entre les princes des Pays-Bas par cet excursus : " Roy de Gades ce Gerrion estoit/Des troys testes dont il est mention,/C'est à cause que deux frères avoit,/Eulx troys estans tous d'une opinion,/Aussi d'un veul vivant en union ${ }^{36}$. Ces petits subterfuges s'étendent en outre aux noms des personnages - le dragon gréco-latin qui défend à Hercule l'entrée du jardin des Hespérides s'appelle d'emblée Maxile, là où attendrait $\operatorname{Ladon}^{37}$, pour faire place à Maximilien, le dragon germanique.

Conscient d'avoir affaire à un héros d'une lointaine antiquité, l'auteur s'évertue quand même à instaurer une manière de continuité entre les figures du héros, du chevalier et du roi. Hercule n'a plus rien à voir avec le personnage courtois du Recueil des histoires de Troie de Raoul Lefèvre, écrit trente ans auparavant ${ }^{38}$. Il agit seul, porte une massue et une peau de lion. Un vocabulaire relativement fluide ou indéterminé fait coïncider les mondes, cependant. Presque incidemment, Hercule redevient un chevalier face au cerf, par exemple : "Quant Herculles en eust ouy parler,/Comme vaillant chevalier de bon zéle,/ Devers le cerf eust grant vouloir d'aller " et se consume sur le bûcher de l'Oeta (sans jeu de mots) en "très chévaléreux " ${ }^{39}$. Hercule et Henri communiquent par le langage universel de la gloire qu'ils acquièrent ${ }^{40}$ - et par l'interversion subreptice de leurs positions : "Juno, plaine d'envye et de malice,/Invitoit fort le roy Euristeus/Qu'il commandast œuvre non pas propice/A Herculles, pour

34 Les Douze triomphes, op. cit., p. 134.

35 Les Douze triomphes, p. 137.

36 Les Douze triomphes, p. 144.

37 Les Douze triomphes, p. 148. Les Argonautiques d'Apollonios de Rhodes mentionnaient Ladon.

38 Raoul Lefèvre, Le Recoeil des Histoires de Troyes, M. Aeschbach (ed.), Berne/Francfort/New York : Peter Lang, «European University Studies, Series XIII », 1987.

39 Les Douze triomphes, p. 139 et 151.

40 Les Douze triomphes, p. 140 et 143-144. 
qui ne regnast plus " ${ }^{41}$. L'un et l'autre " conquièrent " transitivement, sans plus de distinction, les monstres, les ennemis, les batailles - le mot et ses variantes, moins précis que tuer ou occire, taisent l'inachèvement de plusieurs travaux de Henri.

Hercule a accompli douze travaux méritoires, mais la comparaison doit tourner à l'avantage du roi d'Angleterre pour devenir opérante. Le poème se garde bien de revenir sur la naissance du demi-dieu, qui l'emporte de très loin sur celle du moins que rien qu'est Henri. Ce dernier se fait roi par ses exploits; toute autre source de légitimité, comme l'aval du Parlement ou le mariage avec la fille d'Édouard IV, ne saurait être invoquée sans risque. Encore faut-il que ces exploits trouvent un équivalent : quand l'auteur se laisse emporter par son érudition, en racontant l'aventure des serpents étouffés par Hercule au berceau, la comparaison tourne court et il faut embrayer sur les douze triomphes ${ }^{42}$. Supérieur par ses origines, volontairement oubliées, Hercule le cède à Henri en termes de vertu. Sa fin édifiante est rappelée en détail : mortellement blessé par les flèches empoisonnées du héros alors qu'il avait tenté de lui ravir son épouse, Déjanire, le centaure Nessus conseille à celle-ci de remettre en cadeau sa chemise à Hercule pour s'assurer définitivement de sa fidélité. Crédule, Déjanire, inquiète de la passion de son mari pour Iole, fait revêtir à Hercule la tunique empreinte du sang de Nessus et du poison de l'hydre, ce qui lui cause une douleur insupportable et le force à se donner la mort. Pour avoir brisé les liens du mariage, Hercule est donc puni exemplairement par la justice divine ${ }^{43}$. Rien de tel, cela va de soi, avec le bon roi, dénué de vice et grand amateur de vertu et donc, pour ce motif même, hors de portée des mauvais tours d'Envie ${ }^{44}$.

\section{III) LES TRAVAUX INTERMINABLES DE L'USURPATEUR}

La puissance du monarque s'explique donc moins par sa surhumanité, son endurance ou son ingéniosité que par le secours divin qu'il a gagné en pratiquant la vertu et en se couvrant des vertus royales, la seconde peau du lion de Némée, que représentent force, prudence et richesse ${ }^{45}$. Tout au long du poème, la faveur de Dieu, garante d'invulnérabilité, est rappelée sous forme de proverbes ( Nul ne peut nuire à qui Dieu veut aider " ou "Ce que Dieu garde est toujours bien gardé $\left.{ }^{46}\right)$ ou dans le système commandé par le poème lui-même. Hercule a reçu du berger Molorcus sa massue légendaire ; Henri a Dieu lui-même pour pasteur, et la victoire miraculeuse de Bosworth, à laquelle l'armée du Tudor s'est présentée

41 Les Douze triomphes, p. 134.

42 Les Douze triomphes, p. 135.

43 Les Douze triomphes, p. 149-151.

44 Les Douze triomphes, p. 151-152.

45 Les Douze triomphes, p. 136.

46 Les Douze triomphes, p. 133 et 145. 
en nette infériorité numérique, n'a pas d'autre cause ${ }^{47}$. La fortune du roi est un don de grâce pour paraphraser un vers de notre texte.

Hélas ! à vouloir trop bien faire, celui-ci prend des accents angoissants et reflète une réalité rien moins que pacifiée. Les appels au roi et au peuple, qui s'entrecroisent, laissent deviner la lassitude devant la prolongation des troubles et des incertitudes. À la fin du neuvième triomphe, Henri est invité à prendre foi en Dieu, qui l'a aidé parmi tant d'adversités, à ne pas l'oublier ${ }^{48}$. Un peu plus loin, quand sont punis les capitaines, il est écrit que les traîtres reçoivent le mal pour le mal. Aussi le peuple d'Angleterre doit-il rester fidèle au noble roi Henri. Mais qui a dit que la tentation de l'abandonner l'avait saisi ${ }^{49}$ ? La fin de l'œuvre se veut rassurante : le roi doit conserver patience et espoir, croire les poètes que l'on disait autrefois prophètes, maintenir sa confiance en Dieu. Que le peuple prie pour lui, qu'il ait longue vie et que sa descendance règne en paix et glorieusement ${ }^{50}$. Voilà des passages lourds de sous-entendus quant à la tentation du désespoir après pratiquement un demi-siècle d'une guerre civile à laquelle Henri VII n'a pas apporté un terme, malgré ses promesses, malgré ses victoires, malgré la réconciliation apparente des deux roses de Lancastre et d'York. L'usurpateur tire son droit de ses succès et de son bon gouvernement. Or, l'un et l'autre tardent à se confirmer.

Douze travaux pour circonscrire la première moitié du règne, voilà qui est beaucoup et peu à la fois. Le monde du poète est enchanté à plus d'un titre, car bien d'autres conspirations ou contrariétés auraient pu être versées au dossier. Dès la première année du règne, le soulèvement du vicomte Lovell ou, tout près de la date de rédaction du poème, la fronde anti-fiscale qui sévit en Cornouailles sont deux simples exemples qui auraient pu être retenus ${ }^{51}$. Pire, les épreuves mentionnées sont analogues aux têtes de l'hydre et repoussent sans cesse. Les travaux demeurent inaboutis, les triomphes à venir. Que trameront encore Warbeck et les ennemis de l'intérieur ? La douairière, l'empereur et l'archiduc s'avoueront-ils vaincus ? L'Irlande ne reste-t-elle pas, pour l'essentiel, à pacifier, voire, pour reprendre le vocabulaire ambivalent du poème, à conquérir ? L'alliance française sera-t-elle aussi durable que peut le souhaiter un poète français ? La versatilité de la conjoncture internationale n'est déjà plus à prouver. Que le roi patiente ! Et l'auteur de convoquer fort maladroitement l'issue toujours retardée de la première guerre civile romaine : Marius mourut, puis 300000 des siens, et pourtant,

47 Les Douze triomphes, p. 135.

48 Les Douze triomphes, p. 145.

49 Les Douze triomphes, p. 148.

50 Les Douze triomphes, p. 152-153.

51 J. M. Williams, "The political career of Francis Viscount Lovell (1456-?) ", dans The Ricardian, 8:109, (1990), p. 382-402. I. ARTHuRson, "The rising of 1497: a revolt of the peasantry? ", dans J. Rosenthal et C. Richmond (eds.) : People, Politics and the Community in the Later Middle Ages, Gloucester : Sutton, 1987, p. 1-18. 
Sylla dut encore attendre sa paix. Un jour, le roi viendra au bout de sa guerre et aura la victoire ${ }^{52}$. Savant comme il l'était, l'homme de lettres devait être conscient du fait que les douze travaux qui furent sa matière n'étaient que des aventures parmi d'autres pour Hercule - au siècle précédent, Boccace en avait répertorié 31 ! Mais il avait raison sur un point ou deux : le poète, même aveugle, peut se faire voyant, et le chemin de la tranquillité serait encore long pour Henri VII, excédant même les douze années qu'il lui restait à régner. Si Marguerite et Maximilien se rangèrent, si l'alliance française tint, Warbeck fit encore parler de lui en débarquant et en marchant sur Londres à la tête de 6000 hommes, avant d'être capturé et d'intriguer encore avec d'autres leaders yorkistes. Sa mort ne régla pas tous les problèmes. Si l'on en croit Thomas More, la dernière décennie de Henri VII fut un hiver perpétuel, marqué par le soupçon et la répression ${ }^{53}$.

\section{Conclusion}

Pièce de circonstance, les Douze triomphes obéissent aux règles d'un jeu, d'une bagatelle bien de son temps, à propos de laquelle le créateur entend se faire pardonner, comme convenu, aux deux extrémités du poème, sa rhétorique grossière et son simple entendement $t^{54}$.

Il s'agit avant tout d'une forme de discours à la mode, illustrée certes plus fréquemment en latin par le grex poetarum, le troupeau ou disons la petite troupe de flagorneurs internationaux, plumes mercenaires parmi lesquelles Bernard André tient son rang, malgré la concurrence redoutable des humanistes italiens ${ }^{55}$. Le roi a donné une impulsion décisive au phénomène de la cour en Angleterre et la première mission assignée à ses serviteurs est de mettre leur nouveau langage ou leur langage rénové au service de sa douteuse légitimité. Le texte qui m’a intéressé visait-il un public aristocratique anglais ou français ? Sa conservation dans un manuscrit unique ne permet pas de le déterminer. Quoi qu'il en soit, il montre à sa façon que la légitimité des princes mal nés se confond avec celle des héros et leur biographie commune avec une succession d'épreuves. La tâche usante et pour tout dire titanesque prescrite au récent pouvoir de Henri VII l'assimile toutefois moins à Hercule qu’à Sisyphe.

52 Les Douze triomphes, p. 152.

53 Sur la paranoïa nécessaire de l'usurpateur, voir G. Lecuppre, "Le tyran et la peur du complot dans l'Angleterre du XVe siècle ", dans C. Leveleux-Texeira et B. Ribémont (ed.), Le Crime de l'ombre. Complots, conspirations et conjurations au Moyen Âge, Paris : Klincksieck, "Circare ", 2010, p. 135-153.

54 Les Douze triomphes, p. 135 : "Vous, auditeurs, ayes moy excusé,/Se grossement couche ma rhétorique./Comme ignorant je me suis disposé/A ce faire : mon engin si aplicque » et, p. 153 : «En priant tous qui voirres ceste hystoire/Qu'ilz excusent mon simple entendement ".

55 G. Lecuppre, «Henri VII et les humanistes italiens : élaboration d'une légitimité princière et émergence d'un foyer culturel ", dans L. SECCHI-TARUGI (ed.) : Rapporti e scambi tra umanesimo italiano ed umanesimo europeo, Milan : Nuovi Orizzonti, 2001, p. 51-64. 